\title{
Galactic Cosmic Ray increase associated with an interplanetary magnetic cloud observed by HAWC
}

\author{
Alejandro Lara, ${ }^{a, *}$ K. P. Arunbabu ${ }^{a, b}$ and Tatiana Niembro ${ }^{c}$ on behalf of the HAWC \\ Collaboration \\ (a complete list of authors can be found at the end of the proceedings) \\ ${ }^{a}$ Instituto de Geofísica, Universidad Nacional Autónoma de México \\ C.U, Ciudad de México, 04510, México \\ ${ }^{b}$ Department of Physics, St. Paul's College \\ Kalamassery, Kerala, 683503, India \\ ${ }^{c}$ Smithsonian Astrophysical Observatory \\ Cambridge, MA, 02138, USA \\ E-mail: alara@igeofisica.unam.mx
}

We present the observation of an anomalous increment of the galactic cosmic ray (GCR) flux observed by the HAWC array during October 2016. We propose that this anisotropy of the GCR flux was caused by a magnetic flux rope (MFR) i. e., by the helicoidal magnetic field of an interplanetary coronal mass ejection observed at $1 \mathrm{AU}$ at the same time.

We computed the trajectory of protons with energy in the range of 10 to $60 \mathrm{GeV}$, traveling inside the helicoidal magnetic field observed in situ. The direction of these particles changes towards the axis of the MFR resulting in an anisotropy of the GCR flux along this axis.

This model shows that the alignment between the MFR axis and the HAWC's asymptotic direction, combined with the high sensitivity of HAWC, allowed us to observe the effect of the passage of the MFR on the GCR flux.

We present the HAWC observation associated with the passage of the MFR as well as the heliospheric circumstances around such phenomenon.

$37^{\text {th }}$ International Cosmic Ray Conference (ICRC 2021)

July 12th - 23rd, 2021

Online - Berlin, Germany

\footnotetext{
${ }^{*}$ Presenter
} 


\section{Introduction}

The flux of galactic cosmic rays (GCR) is isotropic due to the effect of the magnetic fields that these charged particles encounter between the source and the observer. When these particles reach the inner heliosphere, they interact with the solar wind as described by the Parker equation [1]. The 22 year cycle and Forbush decreases [2,3] are well known examples of the effects of this interaction and have been observed and studied for long time. Although, there is an interesting phenomenon which has been less studied but that deserves more attention: the increments of the GCR flux observed around the time when an interplanetary coronal mass ejection (ICME) is in the vicinity of the Earth. In this work, we analyze one of this kind of events observed by the HAWC in October 2016 (Sec. 3), and we propose a method to quantify the importance of the increment (Sec. 4).

\section{Cosmic Rays and Magnetic Flux Ropes}

In particular, Forbush decreases are caused by ICMEs [4], which are large plasma structures expelled from the Sun with velocities up to $\sim 3000 \mathrm{~km} \mathrm{~s}^{-1}$ and traveling towards the limits of the heliosphere. Roughly, depending on their velocity, ICMEs are structured in three parts: The shock wave, the sheath, and the ejecta.

In general, it is believed that the shock is sweeping away the low energy GCRs [5] when it is traveling in the interplanetary space, causing in this way the decrease of the observed GCR rate.

The sheath is a turbulent region containing turbulent plasma with enhanced density and magnetic field, which provides an additional obstacle for the GCR propagation that helps in the reduction of the GCR flux. The ejecta is the following region with low density $\left(\sim 10 \mathrm{part} / \mathrm{cm}^{3}\right)$, temperature $\left(\sim 10^{5} \mathrm{~K}\right)$ and strong magnetic field $(\sim 10 \mathrm{nT})$ and constitutes a large magnetic obstacle for the GCR flux.

In some cases, the magnetic field inside the ejecta has a very well-defined helicoidal topology known as magnetic cloud and/or magnetic flux rope (MFR). The magnetic field strength $(\sim 10 \mathrm{nT})$ and the length ( $\sim 0.5 \mathrm{AU})$ of the MFR make possible its interaction with the GCR with energies of tens of $\mathrm{GeV}$, in such a way, that once the isotropic flux of GCR enter the MFR, it is forced by the helicoidal magnetic field to change its direction (depending of the energy) and to travel parallel to the axis of the MFR. Figure 1 shows a sketch of the proposed scenario.

\section{HAWC observation}

The HAWC array is located at high altitude (4100 $\mathrm{m}$ asl) and has a large collecting area (22 $000 \mathrm{~m}^{2}$ ) making it an excellent observatory for space weather studies [6]. In particular, we use the TDC-scaler system of HAWC to study the GCR modulation due to solar activity with high accuracy [6]. An example of the TDC-scaler rates in HAWC showing a strong solar modulation is seen in Figure 2 of [7] (hereafter cited as Paper 1), where the anti-correlation between the solar wind velocity and TDC-scaler rates is evident, and less clear but distinguishable, is the correlation between the TDC-scaler rates and periods of strong magnetic field in the solar wind.

As seen in Figure 3 of Paper 1 and in Figure 2 at the end of October 12, 2016 the solar wind velocity increased from $\sim 350$ to $\sim 440 \mathrm{~km} \mathrm{~s}^{-1}$, the interplanetary magnetic field also showed a 


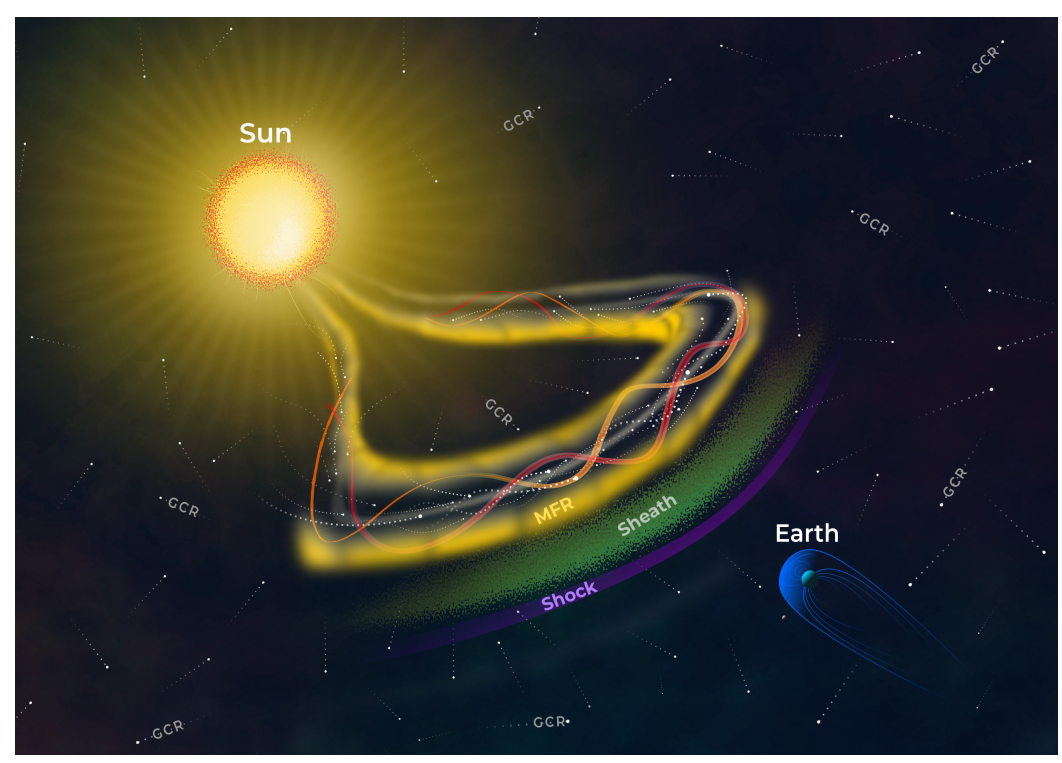

Figure 1: Sketch of the interaction between the galactic cosmic rays and an interplanetary coronal mass ejection approaching the Earth vicinity. The ICME is drivng a shock wave (purple) and the shocked material conforms the sheat (green area) in between the shock and the ejecta. In this case, the former region is a magnetic cloud and its magnetic field with helicoidal topology (reddish lines) guides the GCR (white dots) along the axis of the magnetic flux rope (gray tube).

small increase defining the starting time of an ICME (see Paper 1 for a detailed study of the solar origin and interplanetary transport of this ICME). The associated magnetic cloud or MFR is marked with a shaded area in Figure 2.

The most important feature in our measurements in this period (from October 12 to 15) is the increment of TDC-scaler rate as seen in the second panel of Figure 2, where a double-peak increase of this rate was observed during October 14, 2016.

The helicoidal magnetic field associated with the MFR force the GCR with energies of tents of $\mathrm{GeV}$, to follow a specific direction imposed by the magnetic field topology and causing the alignment of the GCR flux along its axis (see Paper 1 for a detailed explanation of this).

On the other hand, any GCR entering in the magnetosphere experience the Lorentz force, which changes its trajectory to follow an "asymptotic cone of acceptance" which is a function of the energy of GCRs.

We are proposing that the observed double-peak structure in the HAWC TDC-scaler rate data in October 14, 2016 was due to the alignment of this asymptotic cone of observation of HAWC with the MFR axis. The aligned GCRs along the axis of MFR acted like a pseudo-source that created an anisotropic GCR flux.

\section{Significance of the TDC-scaler rates}

After correcting for the atmospheric effects (see [6] for detail), the TDC-scaler rate is largely modulated by the solar wind velocity and also by the atmospheric electric field at the site (this field causes increases in the TDC-scaler rates, see $[8,9]$ ). Hence in order to determine the significance 


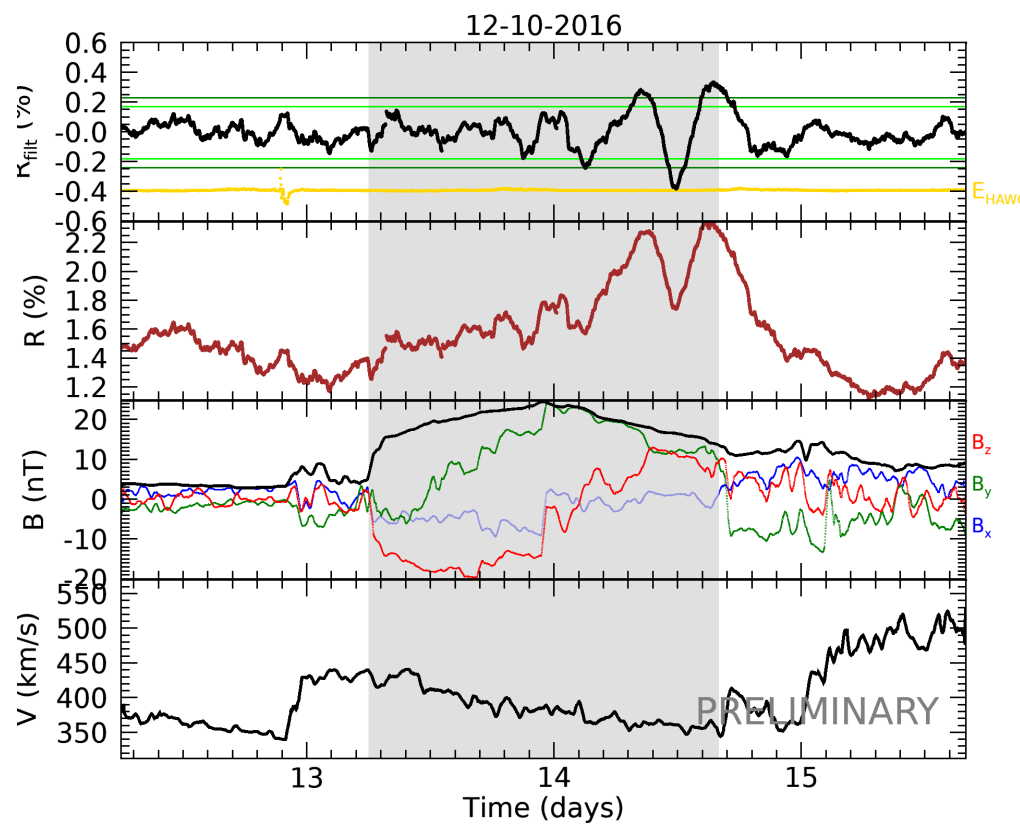

Figure 2: The ICME observed during October 2016. The first panel (from bottom to top) shows the ICME speed, whereas the total strength and components of the magnetic field, showing a helicoidal topology, are plotted in the second panel. The third panel shows the original TDC-scaler rate during this period. The filtered time series is plotted in the fourth panel with the light and dark green lines marking the $3 \sigma$ and $4 \sigma$ confidence levels. In this panel, we also show the electric field, which is scaled to fit the window as $E / 50-0.04$. -the shaded area marks the time duration of the magnetic cloud.

of the modulation caused by the MFR, we filtered out both effects, the long term solar wind velocity modulation and the increment caused by the local electric field. For the latter, we consider the atmospheric electric field which is constantly measured at the site [8,9], which makes possible to identify the periods of strong electric field disturbances. Therefore, by discarding the time periods when both, the local electric field and the TDC-scaler rate are disturbed, we assure that the TDC-scaler rate variations are due to solar wind modulations.

The yellow curve in the top panel of Figure 2 shows the local electric field $(E$, scaled as $E / 50-0.04 \mathrm{kV} / \mathrm{m}$, to fit the window) local electric field strength during the analyzed period, when $E$ was quiet except for a short disturbance on October 12, 2016 at $\sim 22 \mathrm{hr}$.

The details of the MFR and the related TDC-scaler rate increase can be found in Paper 1, in this work, we describe the method proposed to asses the significance of the TDC-scaler rate increases.

In general, the changes in the GCR rate due to the solar wind modulation are longer than one day (as instance, see Figure 2 of Paper 1), on the other hand, the GCR increases, due to the MFR, are affected by the Earth rotation (the axis of the MFR must be parallel to the asymptotic cone of acceptance of the detector [7]) and therefore, its duration is less or equal to one day.

Therefore, to quantify the confidence level of this effect, we compute a GCR long-period time-series using a moving average ${ }^{1}$ with a cut off of one day [10], which is subtracted from to the original time series. In this way, we obtain a filtered time series of the TDC-scaler rate which retains

${ }^{1}$ This is a simple filter in the time domain. 


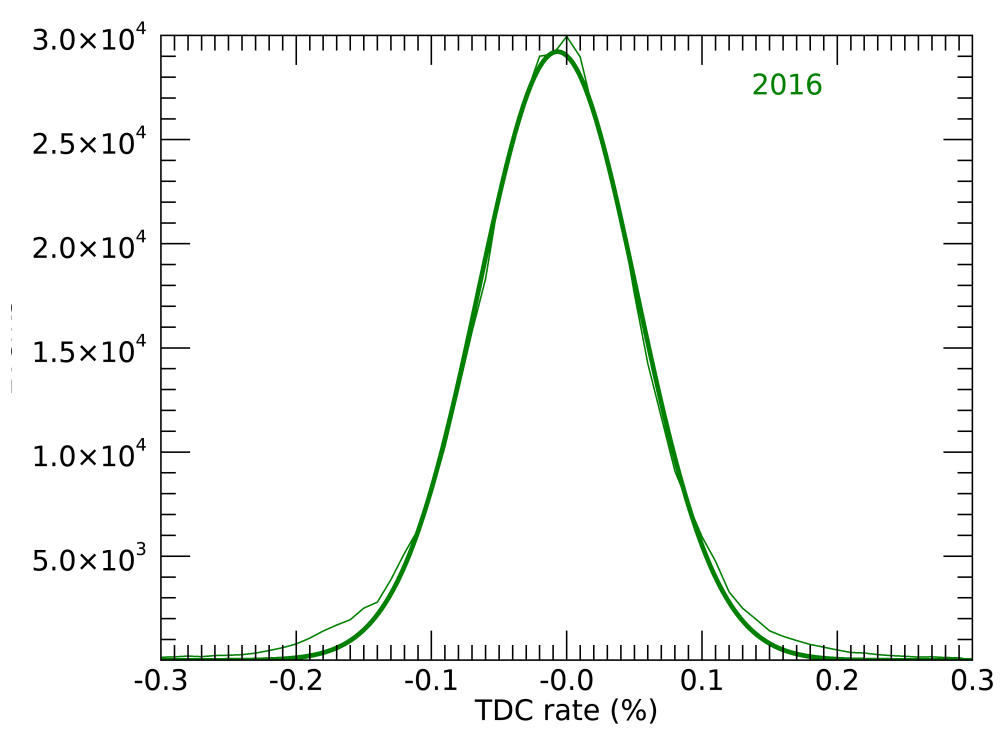

Figure 3: Distribution of the filtered time series of the TDC-scaler rate during the year 2016, the thin green line shows the observed data whereas the thick line, the fitted gaussian. The shaded area corresponds to the fourth sigma confidence region.

only changes with periods shortest than one day, as instance, we show the original TDC-scaler rate series in the second panel of Figure 2, whereas the filtered time series is plotted in the first panel.

Once we have filtered out the long-period fluctuations, it is possible to quantify the importance of the short-period fluctuations, in terms of the standard deviation $(\sigma)$ of the filtered time series distribution which is shown in Figure 3.

With this analysis, we are able to quantify the significance of the TDC-scaler rate increases. In particular, we found that the increase observed during October 2016 has a significance of $\sim 5.7 \sigma$.

\section{Conclusion}

We have shown that the TDC-scaler system of HAWC is an excellent tool to observe and study space weather disturbances, as the MFR observed during October 2016. In addition, in this work, we have proposed a method to quantify the significance of the increases measured with the HAWC TDC-scaler rate. In future works, we will apply this method to quantify the significance of the scaler system data of HAWC taken during the whole period of observations to determine and hence model the effect of MFRs over the GCR flux.

\section{Acknowledgements}

We thank Alejandro Salazar Méndez for the creation Figure 1. We acknowledge the support from: the US National Science Foundation (NSF); the US Department of Energy Office of High-Energy Physics; the Laboratory Directed Research and Development (LDRD) program of Los 
Alamos National Laboratory; Consejo Nacional de Ciencia y Tecnología (CONACyT), México, grants 271051, 232656, 260378, 179588, 254964, 258865, 243290, 132197, A1-S-46288, A1-S22784, cátedras 873, 1563, 341, 323, Red HAWC, México; DGAPA-UNAM grants IG101320, IN111716-3, IN111419, IA102019, IN110621, IN110521; VIEP-BUAP; PIFI 2012, 2013, PROFOCIE 2014, 2015; the University of Wisconsin Alumni Research Foundation; the Institute of Geophysics, Planetary Physics, and Signatures at Los Alamos National Laboratory; Polish Science Centre grant, DEC-2017/27/B/ST9/02272; Coordinación de la Investigación Científica de la Universidad Michoacana; Royal Society - Newton Advanced Fellowship 180385; Generalitat Valenciana, grant CIDEGENT/2018/034; Chulalongkorn University's CUniverse (CUAASC) grant; Coordinación General Académica e Innovación (CGAI-UdeG), PRODEP-SEP UDG-CA-499; Institute of Cosmic Ray Research (ICRR), University of Tokyo, H.F. acknowledges support by NASA under award number 80GSFC21M0002. We also acknowledge the significant contributions over many years of Stefan Westerhoff, Gaurang Yodh and Arnulfo Zepeda Dominguez, all deceased members of the HAWC collaboration. Thanks to Scott Delay, Luciano Díaz and Eduardo Murrieta for technical support.

\section{References}

[1] E.N. Parker, The passage of energetic charged particles through interplanetary space, Planet. Space Sci. 13 (1965) 9.

[2] M.S. Potgieter, Solar Modulation of Cosmic Rays, Living Reviews in Solar Physics 10 (2013) 3.

[3] S.E. Forbush, On the Effects in Cosmic-Ray Intensity Observed During the Recent Magnetic Storm, Physical Review 51 (1937) 1108.

[4] K.P. Arunbabu, H.M. Antia, S.R. Dugad, S.K. Gupta, Y. Hayashi, S. Kawakami et al., High-rigidity Forbush decreases: due to CMEs or shocks?, A\&A 555 (2013) A139.

[5] G. Wibberenz, J.A. Le Roux, M.S. Potgieter and J.W. Bieber, Transient Effects and Disturbed Conditions, SSR 83 (1998) 309.

[6] C. Alvarez, J.R. Angeles Camacho, J.C. Arteaga-Velázquez, K.P. Arunbabu, D. Avila Rojas, V. Baghmanyan et al., HAWC as a Ground-Based Space-Weather Observatory, Solar Physics 296 (2021) 89.

[7] S. Akiyama, R. Alfaro, C. Alvarez, J.R. Angeles Camacho, J.C. Arteaga-Velázquez, K.P. Arunbabu et al., Interplanetary Magnetic Flux Rope Observed at Ground Level by HAWC, ApJ 905 (2020) 73.

[8] A. Lara, G.B. de Raga and O. Enríquez-Rivera, Hawc response to atmospheric electricity activity, 2017.

[9] A.R. Jara Jimenez, A. Lara, K.P. Arunbabu, J. Ryan and HAWC Collaboration, Effects of the atmospheric electric field on the HAWC scaler rate, in 36th International Cosmic Ray 
Conference (ICRC2019), vol. 36 of International Cosmic Ray Conference, p. 1087, July, 2019.

[10] S.W. Smith, Digital Signal Processing, Elsevier Inc. (2002). 


\section{Full Authors List: HAWC Collaboration}

A.U. Abeysekara ${ }^{48}$, A. Albert ${ }^{21}$, R. Alfaro ${ }^{14}$, C. Alvarez ${ }^{41}$, J.D. Álvarez ${ }^{40}$, J.R. Angeles Camacho ${ }^{14}$, J.C. Arteaga-Velázquez ${ }^{40}$, K. P. Arunbabu $^{17}$, D. Avila Rojas ${ }^{14}$, H.A. Ayala Solares ${ }^{28}$, R. Babu ${ }^{25}$, V. Baghmanyan ${ }^{15}$, A.S. Barber ${ }^{48}$, J. Becerra Gonzalez ${ }^{11}$, E. BelmontMoreno $^{14}$, S.Y. BenZvi ${ }^{29}$, D. Berley ${ }^{39}$, C. Brisbois ${ }^{39}$, K.S. Caballero-Mora ${ }^{41}$, T. Capistrán ${ }^{12}$, A. Carramiñana ${ }^{18}$, S. Casanova ${ }^{15}$, O. Chaparro-Amaro $^{3}$, U. Cotti ${ }^{40}$, J. Cotzomi ${ }^{8}$, S. Coutiño de León ${ }^{18}$, E. De la Fuente ${ }^{46}$, C. de León ${ }^{40}$, L. Diaz-Cruz ${ }^{8}$, R. Diaz Hernandez ${ }^{18}$, J.C. Díaz-Vélez ${ }^{46}$, B.L. Dingus ${ }^{21}$, M. Durocher ${ }^{21}$, M.A. DuVernois ${ }^{45}$, R.W. Ellsworth ${ }^{39}$, K. Engel ${ }^{39}$, C. Espinoza ${ }^{14}$, K.L. Fan ${ }^{39}$, K. Fang $^{45}$, M. Fernández Alonso ${ }^{28}$, B. Fick ${ }^{25}$, H. Fleischhack ${ }^{51,11,52}$, J.L. Flores ${ }^{46}$, N.I. Fraija ${ }^{12}$, D. Garcia ${ }^{4}$, J.A. García-González ${ }^{20}$, J. L. García-Luna ${ }^{46}$, G. García-Torales ${ }^{46}$, F. Garfias ${ }^{12}$, G. Giacinti ${ }^{22}$, H. Goksu ${ }^{22}$, M.M. González ${ }^{12}$, J.A. Goodman ${ }^{39}$, J.P. Harding ${ }^{21}$, S. Hernandez ${ }^{14}$, I. Herzog ${ }^{25}$, J. Hinton ${ }^{22}$, B. Hona ${ }^{48}$, D. Huang ${ }^{25}$, F. Hueyotl-Zahuantitla ${ }^{41}$, C.M. Hui ${ }^{23}$, B. Humensky ${ }^{39}$, P. Hüntemeyer ${ }^{25}$, A. Iriarte ${ }^{12}$, A. Jardin-Blicq ${ }^{22,49,50}$, H. Jhee ${ }^{43}$, V. Joshi ${ }^{7}$, D. Kieda ${ }^{48}$, G J. Kunde ${ }^{21}$, S. Kunwar ${ }^{22}$, A. Lara ${ }^{17}$, J. Lee ${ }^{43}$, W.H. Lee ${ }^{12}$, D. Lennarz ${ }^{9}$, H. León Vargas ${ }^{14}$, J. Linnemann ${ }^{24}$, A.L. Longinotti ${ }^{12}$, R. López-Coto ${ }^{19}$, G. Luis-Raya ${ }^{44}$, J. Lundeen ${ }^{24}$, K. Malone ${ }^{21}$, V. Marandon $^{22}$, O. Martinez ${ }^{8}$, I. Martinez-Castellanos ${ }^{39}$, H. Martínez-Huerta ${ }^{38}$, J. Martínez-Castro ${ }^{3}$, J.A.J. Matthews ${ }^{42}$, J. McEnery ${ }^{11}$, P. Miranda-Romagnoli $^{34}$, J.A. Morales-Soto ${ }^{40}$, E. Moreno ${ }^{8}$, M. Mostafá ${ }^{28}$, A. Nayerhoda ${ }^{15}$, L. Nellen ${ }^{13}$, M. Newbold ${ }^{48}$, M.U. Nisa ${ }^{24}$, R. Noriega-Papaqui ${ }^{34}$, L. Olivera-Nieto ${ }^{22}$, N. Omodei ${ }^{32}$, A. Peisker ${ }^{24}$, Y. Pérez Araujo ${ }^{12}$, E.G. Pérez-Pérez ${ }^{44}$, C.D. Rho ${ }^{43}$, C. Rivière ${ }^{39}$, D. Rosa-Gonzalez ${ }^{18}$, E. Ruiz-Velasco ${ }^{22}$, J. Ryan ${ }^{26}$, H. Salazar ${ }^{8}$, F. Salesa Greus ${ }^{15,53}$, A. Sandoval ${ }^{14}$, M. Schneider ${ }^{39}$, H. Schoorlemmer ${ }^{22}$, J. Serna-Franco ${ }^{14}$, G. Sinnis ${ }^{21}$, A.J. Smith ${ }^{39}$, R.W. Springer ${ }^{48}$, P. Surajbali ${ }^{22}$, I. Taboada ${ }^{9}$, M. Tanner ${ }^{28}$, K. Tollefson ${ }^{24}$, I. Torres ${ }^{18}$, R. Torres-Escobedo $^{30}$, R. Turner ${ }^{25}$, F. Ureña-Mena ${ }^{18}$, L. Villaseñor ${ }^{8}$, X. Wang ${ }^{25}$, I.J. Watson ${ }^{43}$, T. Weisgarber ${ }^{45}$, F. Werner ${ }^{22}$, E. Willox ${ }^{39}$, J. Wood ${ }^{23}$, G.B. Yodh ${ }^{35}$, A. Zepeda ${ }^{4}$, H. Zhou ${ }^{30}$

${ }^{1}$ Barnard College, New York, NY, USA, ${ }^{2}$ Department of Chemistry and Physics, California University of Pennsylvania, California, PA, USA, ${ }^{3}$ Centro de Investigación en Computación, Instituto Politécnico Nacional, Ciudad de México, México, ${ }^{4}$ Physics Department, Centro de Investigación y de Estudios Avanzados del IPN, Ciudad de México, México, ${ }^{5}$ Colorado State University, Physics Dept., Fort Collins, CO, USA, ${ }^{6}$ DCI-UDG, Leon, Gto, México, ${ }^{7}$ Erlangen Centre for Astroparticle Physics, Friedrich Alexander Universität, Erlangen, BY, Germany, ${ }^{8}$ Facultad de Ciencias Físico Matemáticas, Benemérita Universidad Autónoma de Puebla, Puebla, México, ${ }^{9}$ School of Physics and Center for Relativistic Astrophysics, Georgia Institute of Technology, Atlanta, GA, USA, ${ }^{10}$ School of Physics Astronomy and Computational Sciences, George Mason University, Fairfax, VA, USA, ${ }^{11}$ NASA Goddard Space Flight Center, Greenbelt, MD, USA, ${ }^{12}$ Instituto de Astronomía, Universidad Nacional Autónoma de México, Ciudad de México, México, ${ }^{13}$ Instituto de Ciencias Nucleares, Universidad Nacional Autónoma de México, Ciudad de México, México, ${ }^{14}$ Instituto de Física, Universidad Nacional Autónoma de México, Ciudad de México, México, ${ }^{15}$ Institute of Nuclear Physics, Polish Academy of Sciences, Krakow, Poland, ${ }^{16}$ Instituto de Física de São Carlos, Universidade de São Paulo, São Carlos, SP, Brasil, ${ }^{17}$ Instituto de Geofísica, Universidad Nacional Autónoma de México, Ciudad de México, México, ${ }^{18}$ Instituto Nacional de Astrofísica, Óptica y Electrónica, Tonantzintla, Puebla, México, ${ }^{19}$ INFN Padova, Padova, Italy, ${ }^{20}$ Tecnologico de Monterrey, Escuela de Ingeniería y Ciencias, Ave. Eugenio Garza Sada 2501, Monterrey, N.L., 64849, México, ${ }^{21}$ Physics Division, Los Alamos National Laboratory, Los Alamos, NM, USA, ${ }^{22}$ Max-Planck Institute for Nuclear Physics, Heidelberg, Germany, ${ }^{23}$ NASA Marshall Space Flight Center, Astrophysics Office, Huntsville, AL, USA, ${ }^{24}$ Department of Physics and Astronomy, Michigan State University, East Lansing, MI, USA, ${ }^{25}$ Department of Physics, Michigan Technological University, Houghton, MI, USA, ${ }^{26}$ Space Science Center, University of New Hampshire, Durham, NH, USA, ${ }^{27}$ The Ohio State University at Lima, Lima, OH, USA, ${ }^{28}$ Department of Physics, Pennsylvania State University, University Park, PA, USA, ${ }^{29}$ Department of Physics and Astronomy, University of Rochester, Rochester, NY, USA, ${ }^{30}$ Tsung-Dao Lee Institute and School of Physics and Astronomy, Shanghai Jiao Tong University, Shanghai, China, ${ }^{31}$ Sungkyunkwan University, Gyeonggi, Rep. of Korea, ${ }^{32}$ Stanford University, Stanford, CA, USA, ${ }^{33}$ Department of Physics and Astronomy, University of Alabama, Tuscaloosa, AL, USA, ${ }^{34}$ Universidad Autónoma del Estado de Hidalgo, Pachuca, Hgo., México, ${ }^{35}$ Department of Physics and Astronomy, University of California, Irvine, Irvine, CA, USA, ${ }^{36}$ Santa Cruz Institute for Particle Physics, University of California, Santa Cruz, Santa Cruz, CA, USA, ${ }^{37}$ Universidad de Costa Rica, San José, Costa Rica, ${ }^{38}$ Department of Physics and Mathematics, Universidad de Monterrey, San Pedro Garza García, N.L., México, ${ }^{39}$ Department of Physics, University of Maryland, College Park, MD, USA, ${ }^{40}$ Instituto de Física y Matemáticas, Universidad Michoacana de San Nicolás de Hidalgo, Morelia, Michoacán, México, ${ }^{41}$ FCFM-MCTP, Universidad Autónoma de Chiapas, Tuxtla Gutiérrez, Chiapas, México, ${ }^{42}$ Department of Physics and Astronomy, University of New Mexico, Albuquerque, NM, USA, ${ }^{43}$ University of Seoul, Seoul, Rep. of Korea, ${ }^{44}$ Universidad Politécnica de Pachuca, Pachuca, Hgo, México, ${ }^{45}$ Department of Physics, University of Wisconsin-Madison, Madison, WI, USA, ${ }^{46}$ CUCEI, CUCEA, Universidad de Guadalajara, Guadalajara, Jalisco, México, ${ }^{47}$ Universität Würzburg, Institute for Theoretical Physics and Astrophysics, Würzburg, Germany, ${ }^{48}$ Department of Physics and Astronomy, University of Utah, Salt Lake City, UT, USA, ${ }^{49}$ Department of Physics, Faculty of Science, Chulalongkorn University, Pathumwan, Bangkok 10330, Thailand, ${ }^{50}$ National Astronomical Research Institute of Thailand (Public Organization), Don Kaeo, MaeRim, Chiang Mai 50180, Thailand, ${ }^{51}$ Department of Physics, Catholic University of America, Washington, DC, USA, ${ }^{52}$ Center for Research and Exploration in Space Science and Technology, NASA/GSFC, Greenbelt, MD, USA, ${ }^{53}$ Instituto de Física Corpuscular, CSIC, Universitat de València, Paterna, Valencia, Spain 